\title{
An Approach to the $A b$ Initio Molecular Orbital Calculation of the Stabilizing Energy on Polymer Double-Stranded Helix
}

\author{
Hiroshi Kusanagi, Yozo Chatani,* and Hiroyuki Tadokoro* \\ Unitika Research Laboratories Inc., Uji, Kyoto 611, Japan \\ * Department of Macromolecular Science, Faculty of Science, Osaka University, \\ Toyonaka, Osaka 560, Japan
}

(Received July 8, 1996)

\begin{abstract}
An energy calculation procedure by using the ab initio molecular orbital (MO) method was derived for the estimation of the stabilizing energy of double-stranded helices on large oligomers and so polymers, although the usual ab initio MO method can not treat polymers. This procedure was applied to the main-chain atom model of isotactic poly(methyl methacrylate) double-stranded helix. The results indicate that the calculated stabilizing energy on small oligomers is in good agreement with those by the usual method. Therefore, this procedure was found to be extensively applicable to the $a b$ initio MO studies on the molecular-weight dependency of the stabilizing energy of polymer double-stranded helices.

KEY WORDS Energy Calculation / Ab Initio Molecular Orbital / Polymer / Double-Stranded Helix / Stabilizing Energy /
\end{abstract}

Isotactic poly(methyl methacrylate) (it-PMMA) has known to take the $(10 / 1)$ double-stranded helix in the crystal. ${ }^{1}$ It would seem to be important to clarify the structural reasons why it-PMMA does form the doublestranded helix, although there is no strong interactions between the two chains in the double-stranded helix, such as, for example, hydrogen bonding in the doublestranded helices of deoxyribonucleic acid (DNA) ${ }^{2,3}$ and poly(ethyleneimine) (PEI). ${ }^{4}$ In a previous paper, an $a b$ initio molecular orbital (MO) calculation was made to evaluate the energetical stability of the double-stranded monomer model of it-PMMA as a model compound for polymer double-stranded helix. ${ }^{5}$ And the monomer model compound was found to be stabilized with the van der Waals interactions. That is, when the 4-31G basis set and the SCF-MO method with the 2nd order Moeller-Plesset (MP2) perturbation theory were adopted, the calculated total MO energy of the doublestranded monomer model gave the stabilizing energy (=the molecular complex formation energy) of $\Delta E=$ $-0.26 \mathrm{kcal} \mathrm{mol}^{-1}$ of monomer, in comparison with twice of the isolated monomer. And the ab initio MO method reveals a strong theoretical proof that the double-stranded helix structure of it-PMMA is correct. But, this monomer model is too small and not enough to consider polymer model. Since the $a b$ initio MO method has a serious limitation on the number of involving atoms and basis functions, the calculation on appropriate large oligomer models is impossible. Figure 1 shows a relation between the computational time (CPU time) and the number of basis functions (NF) in the $a b$ initio MO calculations of several model compounds. From this figure, it is easily seen that the CPU time increases logarizumically with the number of basis functions. In deed, CPU time of the calculation on the above monomer double-stranded helix $(\mathrm{NF}=166)$ of PMMA was 8 hours. But, if we will try the calculation on the pentamer double-stranded helix $(\mathrm{NF}=468)$ of PMMA denoted in the figure, it takes three years. This means that even the calculation on pentamer model is really impossible. Moreover, even if long CPU time is acceptable, there is an another limitation on the number of basis functions, due to the capacity of CPU memory.

Therefore, in the present study we aimed to develop an approach method for the ab initio molecular orbital calculation of the stabilizing energy on polymer doublestranded helix.

\section{METHOD}

The present ab initio MO method concerned with the stabilizing energy on polymer double-stranded helix refers the molecular mechanics (MM) energy calculation method for the single helix of polymers. Therefore, at first, this method is described.

\section{Energy Calculation of Single Helices by Molecular Mechanics}

The MM energy calculation method for helical polymers was developed by Scott and Scheraga, and extensive conformational analyses of polypeptides and related compounds have been made by them. ${ }^{6-8}$ And the

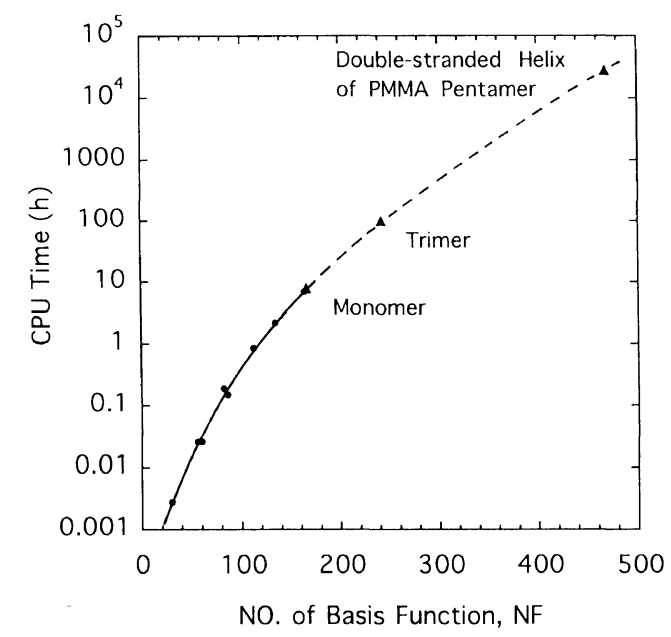

Figure 1. Relation between the computational time (CPU time) and the number of basis functions (NF) in the $a b$ initio $\mathrm{MO}$ calculations on several model compounds. 


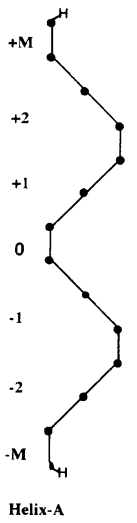

(a)

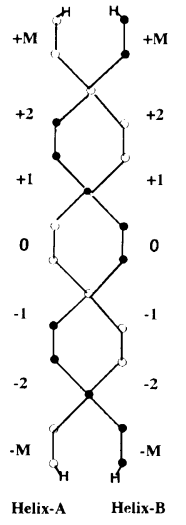

(b)

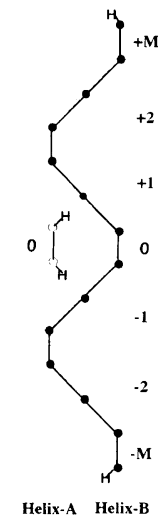

(c)

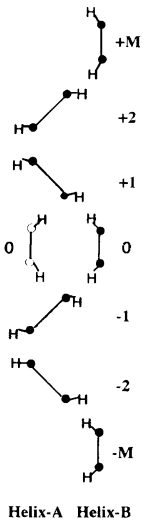

(d)
Figure 2. Schematic representation of helix models used in several energy calculation procedures. (a) Single helix model used in the MM method for calculating the intramolecular interaction energy of single helix. (b) Double-stranded helix model used in the usual MO method for calculating the stabilizing energy of double-stranded helix. (c) Model used in the usual effective MO method. (d) Divided helix model used in the present MO method for double-stranded helix.

method was applied to the synthetic polymers by the Hasegawa and Kobayashi. ${ }^{9}$ Calculation method is as follows. Assuming a helical polymer, represented by Helix-A, which consists of $2 \mathrm{M}+1$ monomer units $(-\mathrm{M}$, $-\mathrm{M}+1, \cdots,-1,0,1, \cdots, \mathbf{M}-1, \mathbf{M})$ (Figure 2a), the intramolecular potential energy per monomer unit, $E_{\text {intra }}$ $(u)$ is written by the equation;

$$
\begin{aligned}
E_{\text {intra }}(u) & =[1 /(2 \mathrm{M}+1)] E_{\text {intra }} \\
& =[1 /(2 \mathrm{M}+1)]\left[(1 / 2) \sum \sum \varepsilon_{\mathrm{A}}(i, j)\right] \\
& =(1 / 2) \sum \varepsilon_{\mathrm{A}}(0, k)
\end{aligned}
$$

where $E_{\text {intra }}$ is the total intramolecular interaction energy of Helix $\mathrm{A}$, and $\varepsilon_{\mathrm{A}}(i, j)$ is the interaction energy between the $i$-th and $j$-th monomer units in Helix-A for $i \neq j$. That is, the intramolecular interaction energy $E_{\text {intra }}(u)$ can be obtained by the single summation from the 0 -th monomer unit to $k$-th monomer unit among the inter-monomer interaction energy $\varepsilon_{\mathrm{A}}(0, k): k=-\mathrm{M}, \cdots 0 \cdots+\mathrm{M}$.

\section{The Usual Ab Initio MO Method for Calculating the Stabilization Energy of Double-Stranded Helices}

In order to estimate the stabilizing energy of doublestranded helices by using the ab initio MO method, the super molecule composed of two same size oligomer helices are generally used (Figure 2b). ${ }^{10}$ In this case, total atoms and NF increase rapidly with the oligomer size. Therefore, the above procedure used in the MM enegy calculation on single helices is effective in this case. According to the procedure of the single helix, the intermolecular interaction energy ( $=$ the stabilizing energy of double-stranded helices) $E_{\text {inter }}(u)$ can be obtained by treating a super molecule composed of the 0 -th monomer unit in the Helix-A and all monomer units of the Helix-B as shown in Figure 2c.

$$
\begin{aligned}
E_{\text {inter }}(u) & =[1 /(2 \mathrm{M}+1)] E_{\text {inter }} \\
& =(1 / 2)\left(E_{\mathrm{AB}}^{\mathrm{MO}}-\varepsilon_{\mathrm{A}}^{\mathrm{MO}}(0)-E_{\mathrm{B}}^{\mathrm{MO}}\right)
\end{aligned}
$$

where $E_{\mathrm{AB}}^{\mathrm{MO}}$ is the $\mathrm{MO}$ energy of the super molecule, $\varepsilon_{\mathrm{A}}^{\mathrm{MO}}(0)$ is the MO energy of the 0 -th monomer in Helix-A, and $E_{\mathrm{B}}^{\mathrm{MO}}$ is the total MO energy of Helix-B. Even if we use eq 2, however, the MO calculation can be made on only small oligomer helices, because of the above limitation on the NF.

\section{The Present Ab Initio MO Method for Calculating the Stabilization Energy of Double-Stranded Helices}

We adopted an idea that Helix-B was divided to monomers with the geometry same as Helix-A. Thereby, a MO calculation procedure was derived by introducing an approximated models as shown in Figure 2c. According to the above procedure of the single helix, the stabilizing energy of double-stranded helices $E_{\text {inter }}(u)$ can be obtained by the single summation from the 0 -th monomer unit in the Helix-A to the $k$-th monomer unit in the Helix-B as shown in Figure 2d.

$$
\begin{aligned}
E_{\text {inter }}(u) & =[1 /(2 \mathrm{M}+1)] E_{\text {inter }} \\
& =[1 /(2 \mathrm{M}+1)]\left[(1 / 2) \sum \sum \varepsilon_{\text {inter }}(i, j)\right] \\
& =(1 / 2) \sum \varepsilon_{\text {inter }}(0, k)
\end{aligned}
$$

where $\varepsilon_{\text {inter }}(0, k)$ is the interaction energy between the 0 -th monomer unit in Helix-A and $k$-th monomer unit in Helix-B. In this method, the energy $\varepsilon_{\text {inter }}(0, k)$ is made individually with each pair of 0 -th monomer unit of Helix-A and $k$-th monomer unit of Helix-B.

$$
\varepsilon_{\text {inter }}(0, k)=\varepsilon_{\mathbf{A B}}^{\mathrm{MO}}(0, k)-\varepsilon_{\mathbf{A}}^{\mathrm{MO}}(0)-\varepsilon_{\mathbf{B}}^{\mathrm{MO}}(k)
$$

where $\varepsilon_{\mathrm{AB}}^{\mathrm{MO}}(0, k)$ is the $\mathrm{MO}$ energy of a small super molecule composed of the 0 -th monomer in Helix-A and the $k$-th monomer in Helix-B, and $\varepsilon_{\mathbf{A}}^{\mathrm{MO}}(0)$ and $\varepsilon_{\mathbf{B}}^{\mathrm{MO}}(k)$ are the MO energies of the 0 -th monomer in Helix-A and of the $k$-th monomer in Helix-B, respectively.

In this procedure, since the MO energy is pairwisely calculated on such as small super molecules of two monomers, the number of involving atoms and NF does not increase with the oligomer size. While, two hydrogen atoms must be added to two end carbon atoms of each monomer (Figure 2d), so that the number of atoms included in the MO calculation is increased. As the result, the intermolecular interactions may also increase someone. This causes to a calculation error in the present MO method. When the molecular weight of monomer unit is large, however, this error may be relatively small. In this paper, the main-chain atom model of PMMA double-stranded helix was calculated and this point was examined.

In order to compare two kinds of calculations by the usual and present MO methods, we used a series of oligomer helices composed of the main-chain atom models $\left(\mathrm{H}-\left(\mathrm{CH}_{2}-\mathrm{CH}_{2}\right)_{n}-\mathrm{H}\right)$, instead of it-PMMA full atom models $\left(\mathrm{H}-\left[\mathrm{C}\left(\mathrm{COOCH}_{3}\right)\left(\mathrm{CH}_{3}\right)-\mathrm{CH}_{2}\right]_{n}-\mathrm{H}\right)$. The main-chain models were constructed by adopting the steric structural parameters determined by X-ray analysis. ${ }^{1}$ The main-chain atom double-stranded helical model of decamer is shown in Figure 3, in comparison with the full atom model.

$A b$ initio MO calculations were carried out with using the version of the Hondo-8 system of programsapplicable maximal number of atoms is 128 . The $4-31 \mathrm{G}$ basis set was adopted, and $a b$ initio SCF-MO method with the electron correlation correction by means of the 2nd order Moeller-Plesset (MP2) perturbation theory was used as the calculation procedure. The present 
Hondo-8 program has been used for MP2 calculations involving up to 250 basis functions $(\mathrm{NF}<250)$. IBM computer work station was used for the calculation.

\section{APPLICATION RESULT AND DISCUSSION}

Table I summarizes calculation results by the usual $a b$ intio MO method. In this method, an applicable maximal oligomer of double-stranded helix model is heptamer $(\mathrm{M}=3)$ with $\mathrm{NF}=216$, and $\mathrm{MO}$ calculations on oligomers more than the heptamer model could not be made. On the other hand, the present $a b$ initio $\mathrm{MO}$ method consists of two calculation steps. In step-1, inter-monomer stabilizing energies $\varepsilon_{\text {inter }}(0, k)$ had been calculated for $k=1,2,3, \cdots \mathrm{M}$, according to eq 4 . The calculated MO energy $\varepsilon_{\mathrm{AB}}^{\mathrm{MO}}(0, k), \varepsilon_{\mathbf{A}}^{\mathrm{MO}}(0), \varepsilon_{\mathbf{B}}^{\mathrm{MO}}(k)$, and inter-monomer stabilizing energy $\varepsilon_{\text {inter }}(0, k)$ were listed in Table II. Next, from these $\varepsilon_{\text {inter }}(0, k)$ values, according
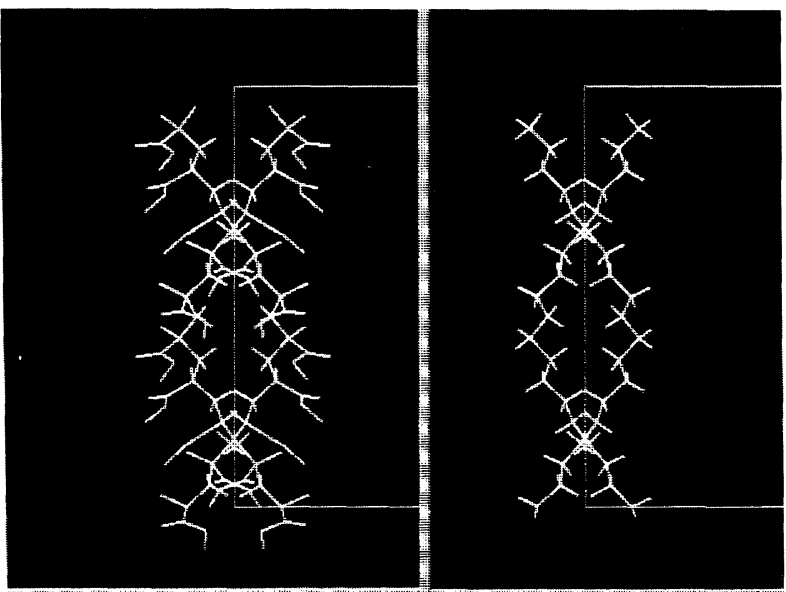

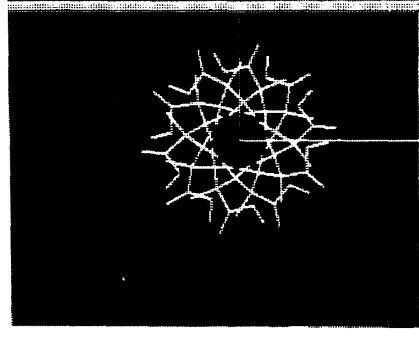

(a)

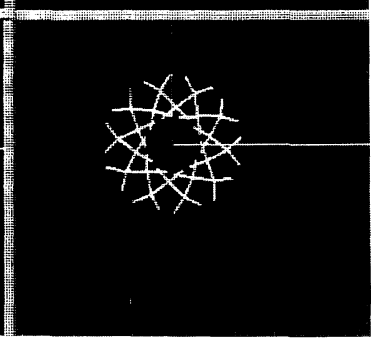

(b)
Figure 3. The main-chain atom model and full atom model of it-PMMA double-stranded helix. (a) Full atom and (b) main-chain atom models. to eq $3, E_{\text {inter }}(u)$ was calculated for each $\mathrm{M}$ value (step-2). In eq 3 , the summation on $k$ was cut with $M=8$, where $\varepsilon_{\mathrm{AB}}^{\mathrm{MO}}(0, k)$ is smaller than $0.0002 \mathrm{kcal} \mathrm{mol}^{-1}$. The calculated stabilizing energy $E_{\text {inter }}(u)$ were listed in Table III, as compared with those by the usual MO method. Two kinds of stabilizing energy $E_{\text {inter }}(u)$ were plotted against the number $n$ of monomers in oligomers as shown in Figure 4. This figure reveals some insights.

At first, the calculated stabilizing energies $E_{\text {inter }}(u)$ on the both methods were found to be in good agreement. The calculated stabilizing energies by the usual MO method are, in whole, about $20 \%$ little than those by the present MO method; $E_{\text {inter }}(u)_{\mathrm{A}}=0.8 E_{\text {inter }}(u)_{\mathrm{B}}$. This discrepancy may be attributed that the difference between the structural models used in both methods. That is, in the calculations by the present MO method, hydrogen atoms were attached to the monomer ends. For an example of $\mathrm{M}=2$, the chemical structure of Helix-B in the former and latter models are $\mathrm{H}-\left(\mathrm{CH}_{2}-\right.$ $\left.\mathrm{CH}_{2}\right)_{5}-\mathrm{H}=\mathrm{C}_{10} \mathrm{H}_{22}$ and $\left(\mathrm{H}-\left(\mathrm{CH}_{2}-\mathrm{CH}_{2}\right)_{1}-\mathrm{H}\right)_{5}=$ $\mathrm{C}_{10} \mathrm{H}_{30}$, respectively, and the ratio of total numbers of atoms in the both models, $32 / 40(=0.8)$, may reflect on the difference of stabilizing energies. Therefore, if the molecular-weight of monomer is large, i.e., in case of it-PMMA full model $\left(\mathrm{H}-\left[\mathrm{C}\left(\mathrm{COOCH}_{3}\right)\left(\mathrm{CH}_{3}\right)-\mathrm{CH}_{2}\right]_{n}-\right.$ $\mathrm{H})$, the discrepancy of the stabilizing energy $E_{\text {inter }}(u)$ may expected to become rather small. Eventually, the present method is considered to be an useful approach for the $a b$ initio MO estimation of the stabilizing energy of double-stranded helix.

Secondary, it was found that with the increasing in number of $n$, the stabilizing energy $E_{\text {inter }}(u)$ increases rapidly at $M=1-5$, and becomes almost to a saturate value of $E_{\text {inter }}(u)=-0.65 \mathrm{kcal} \mathrm{mol}^{-1}$ of monomer unit at $M=5-17$. That is, the large molecular-weight effect for the stabilizing energy is expected in the energy calculations by the $a b$ initio MO method. It may be the first case that the molecular-weight effect in the $a b$ initio MO calculations was found on the model compounds related to the double-stranded helix of it-PMMA.

The stabilizing energy of the full atom model for it-PMMA monomer double-stranded helix ${ }^{5}$ was also plotted in Figure 4. As the result, it may be considered that bulky side groups make a important role for the stabilizing of double-stranded helix of it-PMMA. In comparison of two monomer models, the stabilizing

Table I. Total MO energy ( $\left.E^{\mathrm{MO}}\right)$ and stabilizing enegy $\left(E_{\text {inter }}\right)$ by the usual effective ab initio MO method for the main-chain atom double-stranded helix model of it-PMMA oligomers

\begin{tabular}{|c|c|c|c|c|c|c|c|}
\hline $\begin{array}{l}n^{\mathrm{a}} \\
M^{\mathrm{b}}\end{array}$ & $\begin{array}{c}\text { Monomer } \\
1 \\
0\end{array}$ & $\begin{array}{l}\text { Dimer } \\
2\end{array}$ & $\begin{array}{c}\text { Trimer } \\
3 \\
1\end{array}$ & $\begin{array}{l}\text { Tetramer } \\
4\end{array}$ & $\begin{array}{c}\text { Pentamer } \\
5 \\
2\end{array}$ & $\begin{array}{l}\text { Hexamer } \\
\quad 6\end{array}$ & $\begin{array}{c}\text { Heptamer } \\
7 \\
3\end{array}$ \\
\hline$N F^{\mathrm{d}}$ & 60 & 86 & 112 & 138 & 164 & 190 & 216 \\
\hline$E_{\mathrm{AB}}^{\mathrm{MOe}} / \mathrm{au}$ & -158.60065 & -236.73361 & -314.86704 & -393.00049 & -471.13394 & -549.26694 & -627.39994 \\
\hline$\varepsilon_{\mathrm{A}}^{\mathrm{MOf}} / \mathrm{au}$ & -79.30021 & -79.30021 & -79.30021 & -79.30021 & -79.30021 & -79.30021 & -79.30021 \\
\hline$E_{\mathrm{B}}^{\mathrm{MOg}} / \mathrm{au}$ & -79.30021 & -157.43289 & -235.56592 & -313.70028 & -391.83213 & -469.96673 & -548.09973 \\
\hline$E_{\text {inter }}(u)^{\mathbf{h}} / \mathrm{au}$ & -0.000115 & -0.000255 & -0.000455 & -0.000655 & -0.00080 & -0.000845 & -0.000875 \\
\hline $\begin{array}{l}E_{\text {inter }}(u)^{\mathrm{h}} / \mathrm{kcal} \mathrm{mol}^{-1} \\
\quad \text { of monomer }\end{array}$ & -0.0721 & -0.1605 & -0.2855 & -0.4105 & -0.5015 & -0.5300 & -0.5485 \\
\hline
\end{tabular}

${ }^{\mathrm{a}} n, \mathrm{H}-\left(\mathrm{CH}_{2}-\mathrm{CH}_{2}\right)_{n}-\mathrm{H} .{ }^{\mathrm{b}} \mathrm{M}$, number defined in Figure 2. ${ }^{\mathrm{c}} \mathrm{NA}$, number of total atoms. ${ }^{\mathrm{d}} \mathrm{NF}$, number of total basis functions. ${ }^{\mathrm{e}} E_{\mathrm{AB}}^{\mathrm{MO}}$, total MO energy of a super molecule composed of the 0 -th monomer in Helix-A and all monomers in Helix-B. ${ }^{\mathrm{f}} \varepsilon_{\mathrm{A}}^{\mathrm{MO}}$, MO energy of the 0 -th monomer in Helix-A. ${ }^{\mathrm{g}} E_{\mathrm{B}}^{\mathrm{MO}}, \mathrm{MO}$ energy of Helix-B. ${ }^{\mathrm{h}} E_{\mathrm{inter}}(u)$, the stabilizing energy of double-stranded helix, $E_{\mathrm{inter}}(u)=\left[E_{\mathrm{AB}}^{\mathrm{MO}}-\varepsilon_{\mathrm{A}}^{\mathrm{MO}}-E_{\mathrm{B}}^{\mathrm{MO}}\right]$. 
Table II. Monomer MO energy $\left(\varepsilon^{\mathrm{MO}}\right)$ and inter-monomer stabilizing enegy $\left(\varepsilon_{\text {inter }}\right)$ by the present $a b$ initio MO method for the main-chain atom double-stranded helix model of it-PMMA oligomers

\begin{tabular}{|c|c|c|c|c|c|}
\hline \multirow{2}{*}{$k^{\mathrm{a}}$} & $\varepsilon_{\mathrm{AB}}^{\mathrm{MO}}(0, k)^{\mathrm{b}}$ & $\varepsilon_{\mathrm{A}}^{\mathrm{MO}}(0)^{\mathrm{c}}$ & $\varepsilon_{\mathbf{B}}^{\mathrm{MO}}(k)^{\mathbf{d}}$ & $\varepsilon_{\text {inter }}(0, k)^{\mathrm{e}}$ & $\varepsilon_{\text {inter }}(0, k)^{\mathrm{e}}$ \\
\hline & $\mathrm{au}$ & $\mathrm{au}$ & $\mathrm{au}$ & $\mathrm{au}$ & $\mathrm{kcal} \mathrm{mol}^{-1}$ \\
\hline 0 & -158.600646 & -79.300208 & -79.300208 & -0.000230 & -0.1442 \\
\hline 1 & -158.600887 & -79.300208 & -79.300211 & -0.000468 & -0.2934 \\
\hline 2 & -158.600794 & -79.300208 & -79.300196 & -0.000390 & -0.2445 \\
\hline 3 & -158.600469 & -79.300208 & -79.300199 & -0.000062 & -0.0389 \\
\hline 4 & -158.600442 & -79.300208 & -79.300220 & -0.000014 & -0.0087 \\
\hline 5 & -158.600414 & -79.300208 & -79.300202 & $-0.3810^{-5}$ & -0.0024 \\
\hline 6 & -158.600393 & -79.300208 & -79.300184 & $-0.1310^{-5}$ & -0.0008 \\
\hline 7 & -158.600404 & -79.300208 & -79.300196 & $-0.5010^{-6}$ & -0.0003 \\
\hline 8 & -158.600408 & -79.300208 & -79.300199 & $-0.3010^{-6}$ & -0.0002 \\
\hline
\end{tabular}

${ }^{\mathrm{a}} k$, position number of the paired monomer in Helix-B. $\quad{ }^{\mathrm{b}} \varepsilon_{\mathrm{AB}}^{\mathrm{MO}}(0, k)$, MO energy of a small super molecule composed of the 0 -th monomer in Helix-A and the $k$-th monomer in Helix-B. ${ }^{\mathrm{c}} \varepsilon_{\mathrm{A}}^{\mathrm{MO}}(0)$, MO energy of the 0 -th monomer in Helix-A. ${ }^{\mathrm{d}} \varepsilon_{\mathbf{B}}^{\mathrm{MO}}(k)$, MO energy of the $k$-th monomer in Helix-B. ${ }^{\mathrm{e}} \varepsilon_{\text {inter }}(0, k)$, Inter-monomer stabilizing energy; $\varepsilon_{\text {inter }}(0, k)=\left[\varepsilon_{\mathrm{AB}}^{\mathrm{MO}}(0, k)-\varepsilon_{\mathrm{A}}^{\mathrm{MO}}(0)-\varepsilon_{\mathbf{B}}^{\mathrm{MO}}(k)\right] ; \varepsilon_{\text {inter }}(0, k)=\varepsilon_{\text {inter }}(0,-k)$, because of two -fold rotation symmetry normal to helix axis.

Table III. Stabilizing enegy $\left(E_{\text {inter }}\right)$ of the main-chain atom double-stranded helix models of it-PMMA several oligomers

\begin{tabular}{|c|c|c|}
\hline \multirow{2}{*}{$n^{\mathrm{a}}$} & \multicolumn{2}{|c|}{$E_{\text {inter }}$} \\
\hline & Present MO & Usual MO \\
\hline 1 & 0.0721 & 0.0721 \\
\hline 2 & 0.2188 & 0.1605 \\
\hline 3 & 0.3655 & 0.2855 \\
\hline 4 & 0.4878 & 0.4105 \\
\hline 5 & 0.6100 & 0.5015 \\
\hline 6 & 0.6295 & 0.5300 \\
\hline 7 & 0.6489 & 0.5485 \\
\hline 8 & 0.6533 & - $^{\mathrm{b}}$ \\
\hline 9 & 0.6576 & $-^{\mathbf{b}}$ \\
\hline 10 & 0.6588 & $-^{\mathbf{b}}$ \\
\hline 11 & 0.6600 & $-^{b}$ \\
\hline 12 & 0.6604 & $-\mathbf{b}$ \\
\hline 13 & 0.6608 & $\ldots^{b}$ \\
\hline 14 & 0.6610 & $\ldots$ \\
\hline 15 & 0.6611 & $-^{b}$ \\
\hline 16 & 0.6612 & $-^{b}$ \\
\hline 17 & 0.6613 & $-^{b}$ \\
\hline
\end{tabular}

${ }^{\mathrm{a}} n$, Monomer numbers in oligomers, $\mathrm{H}-\left(\mathrm{CH}_{2}-\mathrm{CH}_{2}\right)_{n}-\mathrm{H}$. ${ }^{\mathrm{b}} \mathrm{Non}$ calculable because of the limitation for NF.

energy, $E_{\text {inter }}(u)=-0.26 \mathrm{kcal} \mathrm{mol}^{-1}$, of the full atom model is greater about 4 times than that value, $E_{\text {inter }}(u)=$ $-0.0721 \mathrm{kcal} \mathrm{mol}^{-1}$, of the main-chain model. The MM energy calculation method has already indicated such a molecular-weight effect in the stabilizing energy of the double-stranded helix of it-PMMA. ${ }^{11}$ Therefore, we will apply the present $a b$ initio MO method to the above full atom double-stranded helix model of it-PMMA, and

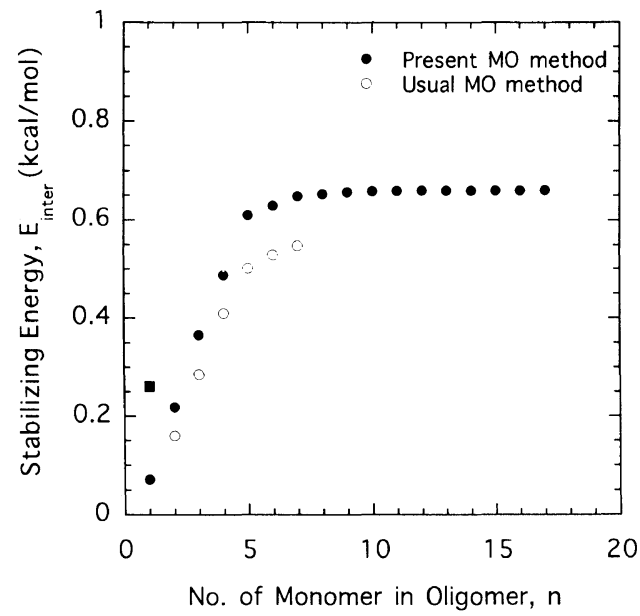

Figure 4. Relation between the stabilizing energy $E_{\text {inter }}(u)$ and the number $n$ of monomers in chain-B for it-PMMA main-chain models. indicates the value for the full atom model of it-PMMA.

compare their results with those by the MM energy calculations.

\section{CONCLUSION}

An energy calculation procedure by using the ab initio MO method was derived for the estimation of the stabilizing energy of double-stranded helices on large oligomers and so polymers, although the usual $a b$ initio MO method can not treat polymers. This procedure was applied to the main-chain atom model of isotactic poly(methyl methacrylate) double-stranded helix. The results indicate that the calculated stabilizing energy is in good agreement with those by the usual method. Therefore, this procedure was found to be extensively applicable to the $a b$ initio MO studies on the molecular weight dependency of the stabilizing energy of polymer double-stranded helices.

\section{REFERENCES}

1. H. Kusanagi, Y. Chatani, and H. Tadokoro, Polymer, 35, 2028 (1994).

2. J. D. Watson and H. C. Crick, Nature (London), 171, 737 (1953).

3. R. Lagrige, H. R. Wilson, C. W. Hooper, and M. H. F. Wilkins, J. Mol. Biol., 2, 19 (1960).

4. Y. Chatani, T. Kobatake, H. Tadokoro, and R. Tanaka, Macromolecules, 15, 170 (1982).

5. H. Kusanagi, Y. Chatani, and H. Tadokoro, Polym. J., 28, 639 (1996).

6. R. A. Scott and H. A. Scheraga, J. Chem. Phys., 42, 2209 (1965).

7. R. A. Scott and H. A. Scheraga, J. Chem. Phys., 44, 3054 (1966).

8. R. A. Scott and H. A. Scheraga, J. Chem. Phys., 45, 2091 (1965).

9. R. Hasegawa, M. Kobayashi, and H. Tadokoro, Polym. J., 3, 591 (1972).

10. H. Kusanagi, Polym. J., 28, 362 (1996).

11. H. Kusanagi, Polym. J., 28, in press. 